\title{
Low peripheral levels of insulin growth factor-1 are associated with high incidence of delirium among elderly patients: A systematic review and meta-analysis
}

Running title: MA of IGF-1 and delirium

Dian-Jeng Li ${ }^{\text {a,b,\# }}$, Ping-Tao Tseng ${ }^{\text {c,\# }}$, Brendon Stubbs ${ }^{\text {d,e,f }}$, Tien-Yu Chen ${ }^{\text {g,h }}$, Pao-Yen Lin $^{\mathrm{i}, \mathrm{j}}$, Shiou-Lan Chen ${ }^{\mathrm{b}}$, Trevor Thompson ${ }^{\mathrm{k}}$, Dimitrios Adamis ${ }^{1, \mathrm{~m}}$, Che-Sheng $\mathrm{Chu}^{\mathrm{n}, \mathrm{o},{ }^{*}}$

${ }^{a}$ Department of Addiction Science, Kaohsiung Municipal Kai-Syuan Psychiatric Hospital, Kaohsiung City, Taiwan

${ }^{b}$ Graduate Institute of Medicine, College of Medicine, Kaohsiung Medical University, Taiwan

${ }^{c}$ WinShine Clinics in Specialty of Psychiatry, Kaohsiung City, Taiwan

${ }^{d}$ Physiotherapy Department, South London and Maudsley NHS Foundation Trust, London, UK

${ }^{e}$ Health Service and Population Research Department, Institute of Psychiatry, Psychology and Neuroscience (IoPPN), King's College London, De Crespigny Park, London, $U K$

${ }^{f}$ Faculty of Health, Social Care and Education, Anglia Ruskin University, Chelmsford, $U K$

${ }^{g}$ Department of Psychiatry, Tri-Service General Hospital; School of Medicine, National Defense Medical Center, Taipei, Taiwan

${ }^{h}$ Institute of Brain Science, National Yang-Ming University, Taipei, Taiwan

${ }^{i}$ Department of Psychiatry, Kaohsiung Chang Gung Memorial Hospital and Chang Gung University College of Medicine, Kaohsiung, Taiwan

${ }^{j}$ Institute for Translational Research in Biomedical Sciences, Kaohsiung Chang Gung Memorial Hospital

${ }^{k}$ Faculty of Education and Health, University of Greenwich, London SE9 2UG 
${ }^{l}$ Sligo Mental Health Services Clarion Rd Sligo, Ireland

${ }^{m}$ Research and Academic Institute of Athens, Greece

${ }^{n}$ Department of Psychiatry, Kaohsiung Veterans General Hospital, Kaohsiung, Taiwan

${ }^{o}$ Center for Geriatric and Gerontology, Kaohsiung Veterans General Hospital, Kaohsiung, Taiwan

" contributed equally as first author

\section{* Correspondence to:}

Che-Sheng Chu, MD

Department of Psychiatry, Kaohsiung Veterans General Hospital, Kaohsiung, Taiwan

Address: No. 386, Dazhong $1^{\text {st }}$ Rd., Zuoying Dist., Kaohsiung City 81362, Taiwan

Telephone: 886-7-3422121

Fax: 886-7-3422288

E-mail: youngtzuchi@hotmail.com/cschu@,vghks.gov.tw 


\begin{abstract}
:
Introduction: Delirium, a serious condition observed in critically ill patients, clinically presents with impaired cognition and consciousness. The relationship between delirium and peripheral levels of insulin growth factor-1 (IGF-1) is unclear. Thus we conducted a meta-analysis to address this issue.
\end{abstract}

Methods: Seven major electronic databases were searched from inception until October 2, 2017 to obtain relevant clinical variables to compare the difference in IGF-1 levels between delirious and non-delirious elderly in-patients. A random effects meta-analysis was conducted.

Results: We studies 10 articles involving 294 delirious patients (mean age 73.0 years) and 604 non-delirious patients (mean age 76.9 years). We found that peripheral levels of IGF-1 in patients with delirium were significantly lower than in those without delirium (Hedges` $g=-0.209,95 \%$ confidence interval $[\mathrm{CI}]=-0.393$ to $-0.026, p=$ 0.025). Meta-regression analyses found that no variables such as percentage of cognitive impairment, mean age, and female proportion contribute to heterogeneity in terms of the entire population.

Conclusions: Our data suggests that lower peripheral levels of IGF-1 could be associated with a higher incidence of delirium among elderly patients. Further 
prospective studies with larger sample sizes are needed to investigate the association between peripheral levels of IGF-1 and delirium.

Keywords: Biomarker, Delirium, Elderly, IGF-1, Meta-analysis 


\section{Introduction}

Delirium, which clinically presents with impairment in cognition, consciousness, psychomotor behavior, and attention (Ely et al., 2001), may manifest with a wide range of subtypes ranging between hyperactive and hypoactive conditions (Steiner, 2011). Clinically, it usually presents with a sudden onset, which then fluctuates between hours and days and is generally a reversible condition (Yudofsky \& Hales, 2008). Etiologically, delirium indicates an organic decline in cerebral function resulting from variable mechanisms. For example, the etiology of alcohol-withdrawal delirium differs from that causing postoperative delirium (Steiner, 2011). Incidence of delirium varies depending on the purpose of investigation. Incidence of delirium varies between $9 \%$ and $43 \%$ among geriatric patients hospitalized for variable medical conditions (Laurila, Pitkala, Strandberg, \& Tilvis, 2002; O'Keeffe \& Gosney, 1997). Delirium is commonly observed in elderly patients post surgery, and the incidence of postoperative delirium (POD) ranges widely between $10 \%$ and $65 \%$ (Radtke et al., 2010; Rudolph \& Marcantonio, 2011). Delirium is a serious neuropsychiatric condition with a substantial impact owing to increased morbidity and mortality, high healthcare costs and prolonged hospitalization associated with it (Hustey, Meldon, Smith, \& Lex, 2003; Meagher et al., 2007). Regarding mortality rates, reportedly, delirious patients show an overall hazard ratio of 1.9 in elderly 
patients in a meta-analysis study (Witlox et al., 2010). Given the inadequate understanding of its multifactorial aetiopathogenesis (Hughes, Patel, \& Pandharipande, 2012), researchers are interested in studying the etiology of delirium to better predict its course and prognosis in clinical settings. Although previous reviews (Khan, Zawahiri, Campbell, \& Boustani, 2011) have demonstrated the limited role of biomarkers in detection of delirium, researchers have been trying to identify specific biomarkers linked to this condition such as insulin growth factor-1 (IGF-1).

IGF-1 is a member of the IGF family with 70 amino acid peptides, and is primarily produced in the central nervous system (CNS) and peripheral tissues such as the liver (Le Roith, 2003; Torres-Aleman, 2010). IGF-1 in the CNS plays a vital role in enhancing neuronal survival, differentiation, synaptogenesis, and recovery from neural injury (D'Ercole, Ye, \& O'Kusky, 2002). IGF-I blocks apoptosis in damaged neurons via several mechanisms, such as delay of apoptosis via activation of PI3K/Akt kinase and Ras/Raf/MEK/ERK pathways and results in long-term survival (Gluckman et al., 1998; Parrizas \& LeRoith, 1997; Peruzzi et al., 1999). Clinically, studies have explored its neuroprotective role and association with neurocognitive disorders such as Alzheimer's disease (AD), of which is involved with delirium (Kooijman, Sarre, Michotte, \& De Keyser, 2009; Lackey, Gray, \& Henricks, 2000). Additionally, IGF-1 deficiency in humans may cause growth abnormalities, including 
a smaller sized brain and mental retardation (Werner \& LeRoith, 2014). Furthermore,

it has been reported that serum IGF-1 levels positively correlate with cognitive

performance in healthy elderly subjects (Al-Delaimy, von Muhlen, \& Barrett-Connor, 2009; Aleman et al., 2000).

Moreover, as researchers keenly investigating the association between peripheral levels of IGF-1 and delirium, several studies have explored the significance of this correlation in patients undergoing surgery or those with acute medical illnesses (Adamis et al., 2009; Adamis et al., 2007; Cerejeira, Batista, Nogueira, Vaz-Serra, \& Mukaetova-Ladinska, 2013; Egberts et al., 2015; Lemstra, Kalisvaart, Vreeswijk, van Gool, \& Eikelenboom, 2008; Morandi et al., 2011; Wilson, Broadhurst, Diver, Jackson, \& Mottram, 2005). While some studies report that lower peripheral levels of IGF-I could significantly increase the incidence of delirium (Cerejeira et al., 2013; Egberts et al., 2015; Morandi et al., 2011; Wilson et al., 2005), the opposite findings have also been reported by some others (Adamis et al., 2009; Adamis et al., 2007; Lemstra et al., 2008). Possible factors accounting for this discrepancy are inconsistency between recruited patients, such as postoperative or medically ill cases in terms of study designs or clinical status. Thus, the association between peripheral levels of IGF-I and incidence of delirium in a clinical setting needs to be further studied. Given these aforementioned gaps existing in literature, we performed a 
comprehensive systematic review and meta-analysis investigating the association between peripheral levels of IGF-1 and delirium, specifically focusing on clinical status of patients. In addition to exploring the difference in this association between surgical or medically ill patients, we also attempted to determine possible factors affecting the correlation between delirium and IGF-1 levels.

\section{Materials and methods}

Our systematic review and meta-analysis was based on Meta-analysis Of

Observational Studies in Epidemiology (MOOSE) guidelines (Stroup et al., 2000) (supplemental table S1) and was approved by the Institutional Review Board of the Tri-Service General Hospital (TSGHIRB: B-105-12). Two investigators (DJ Li and CS Chu) independently performed database searches, study selection, data extraction, and rating of the methodological quality of included studies. Disagreements between investigators were resolved thorough consensus.

\subsection{Database searches}

PubMed, Embase, ScienceDirect, ClinicalKey, Cochrane Library, ClinicalTrials.gov and ProQuest were systematically searched from the time of their 
inception until October $2^{\text {nd }}, 2017$. Keywords used for our search included "(IGF OR

IGF1 protein OR inflammation mediators OR insulin-like growth factor OR cytokine)

AND (delirium OR acute confusion OR consciousness disturbance)" without any

limitation about language. Detailed search strings used for the electronic database

search and results are shown in the supplemental figure S1.

\subsection{Eligibility Criteria and Study Selection}

Eligibility criteria applied were: (1) case-control studies comparing peripheral

IGF-1 levels between delirious patients and non-delirious controls, or cohorts with detailed baseline data and subsequent delirium risk; (2) those with a diagnosis of delirium based on the definite diagnostic systems or scales, such as confusion assessment method (CAM) (Inouye et al., 1990), the diagnostic and statistical manual of mental disorders, $3^{\text {rd }}$ revision (American Psychiatric Association. 1980) and text-revision (DSM-IV-TR) (American Psychiatric Association. 2000), or Delirium observation screening scale (Schuurmans, Shortridge-Baggett, \& Duursma, 2003), Richmond agitation-sedation scale (Sessler et al., 2002); (3) those in whom detailed methods for detecting peripheral IGF-1 were available; and (4) formal registered trials or formal published peer-reviewed articles.

Exclusion criteria were: (1) studies without adequate non-delirious controls; (2) 
articles without relation to the comparisons of peripheral IGF-1 levels in delirious patients and controls; (3) those irrelevant to delirium; (4) review articles/commentary articles/case reports/letter to editor; or (5) non-clinical trials in human.

\subsection{Methodological Quality Assessment}

The modified Newcastle-Ottawa Scale (NOS) was used to rate methodological quality of included studies (Wells GA). This scale considers three domains: selection of participants (maximum of four stars), comparability of groups (maximum of two stars), and measurement (maximum of three stars). Scores ranged from 0 (the lowest) to 9 (the highest), and a score equal to 7 indicated high methodological quality.

\subsection{Data Extraction}

A predetermined data extraction list was used to extract data for this meta-analysis. To be specific, the extracted data included basic characteristics of participants (mean age and percentage of female), mini-mental status examination (MMSE), and duration of hospitalization.

\subsection{Meta-analysis}

The primary outcomes were the difference in peripheral IGF-1 levels in delirious patients compared to control groups who did not develop delirium in each recruited 
study (calculated as Hedges' $g$ statistic). We did not choose difference in means as

effect sizes (ESs) of our primary outcome due to presumed different units used among each study. For studies in which peripheral levels of IGF-1 were not available, outcomes were chosen as odd ratios (ORs) (Morandi et al., 2011; Wilson et al., 2005), or only a $p$ value (Adamis et al., 2007) according to manuals of Comprehensive Meta-Analysis version 3.0. When peripheral levels of IGF-1 were not available in the included studies, we tried to contact the authors of such studies on at least two separate occasions to try and obtain additional raw data. Additionally, we converted ORs into ES using the formula suggested by the Cochrane Handbook (Higgins \& Green, 2011) and derived ES using other statistical parameters such as the $t$ or $p$ value with the sample size when raw data were not available via email inquiry. We defined an ES and Hedges' $g$ less than 0 as "lower peripheral IGF-1 levels in patients with delirium than in controls without delirium". Statistical significance was set as a two-tailed $p$ value $<0.05$. All meta-analytical procedures were performed using the Comprehensive Meta-Analysis version 3 (CMA ver. 3.0) (Biostat, Englewood, NJ) software. Meta-analyses were performed using random-effects models due to the anticipated heterogeneity across studies. Briefly, random-effects modeling is more stringent and therefore more convincing than fixed-effects modeling and incorporates a between-study variance in the calculations (Borenstein, Hedges, Higgins, \& 
Rothstein, 2010).

2.6. Heterogeneity, publication bias, sensitivity test, and meta-regression

We used the Cochran's Q test and the corresponding $p$ value to investigate potential heterogeneity among recruited studies (Higgins \& Thompson, 2002). The $I^{2}$ metric was used to investigate the proportion of variance between recruited studies (Borenstein, Higgins, Hedges, \& Rothstein, 2017). The publication bias was determined by visual examination of funnel plots in situations of less than 10 studies (Higgins \& Green, 2011) and using thorough Egger's regression analysis if there were more than 10 studies (Egger, Davey Smith, Schneider, \& Minder, 1997). In situation of significant publication bias, we arranged Duval and Tweedie`s trim and fill test to adjust the results of meta-analysis (Duval \& Tweedie, 2000). Additionally, we performed sensitivity testing with one study removal test to investigate potential confounding by any one of the outlier in the recruited studies, which had been widely used in meta-analysis (Tobias, 1999). Additionally, we performed meta-regression analysis using an unrestricted maximum likelihood method to explore potential moderators. Variables considered for meta-regression analyses were: mean age, female gender (\%), MMSE score, and a total modified NOS scores. The meta-regression procedure was only performed when moderator variables were 
available from more than 5 individual datasets.

\section{Results \\ 3.1. Studies included in the meta-analysis}

The whole search picture was depicted as figure 1 and supplemental figure S1. In brief, eighty-two articles enter the full-texts investigation stage, and seventy-two were excluded for specific reasons (see supplemental table S2). Finally, ten articles met our inclusion criteria (Adamis et al., 2007; Cerejeira et al., 2013; Chu et al., 2016; Cinar et al., 2014; Egberts et al., 2015; Lemstra et al., 2008; Morandi et al., 2011; Shen, Shao, Chen, \& Guo, 2016; Wilson et al., 2005; Yen et al., 2016). Peripheral levels of IGF-1 were obtained before delirium occurring in the seven studies (Cerejeira et al., 2013; Chu et al., 2016; Cinar et al., 2014; Lemstra et al., 2008; Shen et al., 2016; Wilson et al., 2005; Yen et al., 2016). The participants in other three studies might be drawn IGF-1during delirious status (Adamis et al., 2007; Egberts et al., 2015; Morandi et al., 2011).

\subsection{Methodological quality assessment}

The mean modified NOS score was 7.4 with standard deviation (SD) $=0.5$ across 
the ten studies. The score in each domain of the modified NOS is shown in supplemental table S3.

\author{
3.3. The main results of the meta-analysis comparing the levels of IGF-1 in patients \\ with delirium and those without delirium
}

Our systematic review and meta-analysis included 10 articles that met our inclusion criteria. Peripheral (serum or plasma) IGF-1 levels were measured in these ten studies.

The quantitative determination of IGF-1 were taken from plasma (Cerejeira et al., 2013; Wilson et al., 2005) and serum (Adamis et al., 2007; Chu et al., 2016; Cinar et al., 2014; Egberts et al., 2015; Morandi et al., 2011; Shen et al., 2016; Yen et al., 2016), respectively. One study (Lemstra et al., 2008) mentioned that IGF-1 was assessed from blood sample (table 1). We found 294 delirious patients (mean age 73.0 years, women $51.0 \%$ ) and 604 non-delirious patients (mean age 76.9 years, women $53.2 \%$ ) among the 10 studies. Peripheral levels of IGF-1 in patients with delirium were significantly lower than in those without delirium (Hedges' $g=-0.209,95 \%$ confidence interval $(\mathrm{CI})=-0.393$ to $-0.026, p=0.025]$ (Figure 2) with significant heterogeneity $\left(\mathrm{Q}\right.$ value $=20.215$, degree of freedom $\left.(\mathrm{df})=9, I^{2}=55.479 \%, p=0.017\right)$ but no significant publication bias based on Egger's test $[\mathrm{t}$ value $=1.060, \mathrm{df}=8, p=$ $0.320]$. 


\subsubsection{Sensitivity test}

The significant result of current meta-analysis became non-significant after removal of the datasets by Adamis, D. (2007) (Hedges' $g=-0.184,95 \% \mathrm{CI}=-0.374$ to $0.006, p=0.058$ ) (Adamis et al., 2007), Egberts, A. (2015) (Hedges' $g=-0.175$, 95\% CI $=-0.362$ to $0.011, p=0.066$ ) (Egberts et al., 2015), Shen, H. (2016) (Hedges' $g=-0.125,95 \% \mathrm{CI}=-0.262$ to $0.012, p=0.073$ ) (Shen et al., 2016), or Yen, T.E. (2016) (Hedges' $g=-0.193,95 \% \mathrm{CI}=-0.390$ to $0.004, p=0.055)$ (Yen et al., 2016).

\subsubsection{Meta-regression}

There was no any significant association between the differences of peripheral IGF-1 and clinical variables, including percentage of cognitive impairment ( $p=0.960)$, mean age $(p=0.978)$, female proportion $(p=0.721)$, mean MMSE scores $(p=0.929)$, or quality of methodology as total modified NOS scores $(p=0.270)$.

\section{Discussion}

To the best of our knowledge, the study is the first meta-analysis to address the association between peripheral IGF-1 and delirium and suggests that lower peripheral IGF-1 levels were found in patients with delirium compared with those without 
delirium. Although causality of delirium and peripheral levels of IGF-1 has yet to be clarified, our study offers additional evidence supporting the immune-inflammatory hypothesis in delirium.

The link between delirium and peripheral levels of IGF-1 can be mediated by cytokines released during inflammatory processes. Reportedly, a strong correlation is seen to exist between delirium and inflammation (Broadhurst \& Wilson, 2001).

Peripheral IGF-1 can cross the blood-brain barrier and play a role in neuronal function (Reinhardt \& Bondy, 1994). It has been reported that administration of IGF-1 reduces secondary neuronal loss after transient neural injuries (Gluckman et al., 1998; Trejo, Carro, Garcia-Galloway, \& Torres-Aleman, 2004). Similarly, replacement therapy with IGF-1 ameliorates diabetic neuropathy (Schmidt et al., 1999). Our study provides additional evidence to support this hypothesis. Lower peripheral levels of IGF-1 may possess lesser neuroprotective properties that is anti-apoptotic and anti-inflammatory actions, and contributed higher risk of delirium.

\author{
Another factor involved in the connection between peripheral levels of IGF-1 and \\ delirium may be relatively high mean age (almost above 70 years old) of participants \\ in the included studies. Ageing, resulting from increased natural immunity activity, is \\ a low level inflammatory status (Godbout \& Johnson, 2009; Licastro et al., 2005).
}


Inflammation is related to several morbid conditions in the elderly such as

cardiovascular disease (Pai et al., 2004) or Alzheimer's disease (Casserly \& Topol, 2004). Furthermore, inflammation may result in the breakdown of the blood-brain barrier (Jeppsson et al., 1981) and decrease cholinergic transfer(Hammond, Meador, Aung-Din, \& Wilder, 1987). Taken together, elevated levels of proinflammatory cytokine levels have been suggested to predispose the development of delirium in elderly, which is interpreted by the concept of immunosenescence (Godbout \& Johnson, 2006). IGF-1, involving neuro-inflammation in the CNS (Trejo et al., 2004), may be also affected by ageing, be associated with delirium. However, our meta-regression showed that mean age did not significantly involve the relation between delirium and IGF-1. In addition to limited case numbers, relatively narrowing distribution of mean age may also restrict the statistical significance. Previous study also indicated that older patients undergoing cardiovascular surgery may develop delirium easily (Cinar et al., 2014). However, clearly more research is required to better understand this issue.

It has been reported that IGF-1 levels positively correlate with cognitive performance in healthy elderly subjects (Al-Delaimy et al., 2009; Aleman et al., 2000). A meta-analysis has reported a significantly positive correlation between IGF-I and cognition in a healthy elderly population (Arwert, Deijen, \& Drent, 2005). Another 
study concluded that the IGFI levels showed an inverse correlation with cognitive dysfunction (Murialdo et al., 2001). In the present meta-analysis study, only two studies enrolled participants with baseline cognitive healthy, which was defined as an MMSE score of $\geqq 24$ or excluded when enrollment, and reported that lower peripheral levels of IGF-1 associated with incident of delirium in one study (adjusted Odd ratios $[\mathrm{aORs}]=2.52,95 \% \mathrm{CI}=1.19$ to $5.43, \mathrm{p}=0.019)($ Shen et al., 2016) but not another (aORs $=0.99,95 \% \mathrm{CI}=0.96$ to $1.01, \mathrm{p}=0.183)$ (Yen et al., 2016). For participants with baseline cognitive impairment, six (Adamis et al., 2007; Cerejeira et al., 2013; Chu et al., 2016; Cinar et al., 2014; Lemstra et al., 2008; Morandi et al., 2011) out of eight (Adamis et al., 2007; Cerejeira et al., 2013; Chu et al., 2016; Cinar et al., 2014; Egberts et al., 2015; Lemstra et al., 2008; Morandi et al., 2011; Wilson et al., 2005) studies showed no significant differences between peripheral levels of IGF-1 and delirium. Therefore, cognitive impairment could be a possible confounder unfavorably affecting the association between delirium and peripheral levels of IGF-1. However, our meta-regression showed that baseline MMSE scores were not significantly linked to a correlation between delirium and IGF-1. This difference may be due to variable statistical methodology between dichotomous grouping [normal cognition vs. mild cognitive impairment (MCI) or dementia] and continuous variables of baseline MMSE scores. The fact that a limited number of cases were studied might 
contribute to the insignificance observed with respect to baseline MMSE scores.

Furthermore, some studies report that IGF-I levels were unaltered or increased among dementia patients (Nasman et al., 1995; Sara et al., 1982; Tham et al., 1993). A greater number of studies will need to be performed in future to investigate the correlation between cognition and IGF-1 to explore this inconsistency.

Limitations of our study that need to be addressed are: 1) Studying a limited number of cases might be a confounding factor impacting the significance of outcomes. 2) Higher mean age would restrict the application of our findings to younger adults or adolescents. 3) We could not perform subgroup analyses comparing IGF-1 levels in different delirious classifications or diagnostic criteria (hypoactive vs hyperactive delirium and only CAM vs CAM plus DSM-III or -IV diagnosis) due to not enough studies. Future research is required to understand if this influences the relationship observed. 4) Peripheral levels of IGF-1 do not actually reflect the brain levels, and thus the pathogenesis between CNS levels of IGF-1 and incident delirium need to be addressed in future studies.

\section{Conclusion}

In conclusion, lower peripheral levels of IGF-1 appear to be associated with 
delirium among elderly patients. Further studies involving a greater number of participants and wider ranges of age would help to investigate the etiology and association between these two entities in future. Studies including more baseline variables and more specific description of diagnosing method to delirium could help us investigate more covariates and conduct subgroup analysis. 


\section{Conflict of Interest}

The authors state that there are no any competing interests in the current literature.

\section{Acknowledgements}

The authors declare that there are no conflicts of interest or funding in relation to

the subject of this study. 


\section{Figure legends:}

Figure 1. Flow chart showing the selection strategy and inclusion/exclusion criteria employed in our current meta-analysis.

Figure 2. Forest plot of results of pooled effect sizes (Hedges' $g$ ) and $95 \%$ confidence intervals (CIs) from pooled results of peripheral levels of insulin growth factor-1 (IGF-1) between delirious and non-delirious patients within the entire population.

Abbreviation: CI: confidence interval; IGF-1: insulin-like growth factor-1 


\section{References:}

Adamis, D., Lunn, M., Martin, F. C., Treloar, A., Gregson, N., Hamilton, G., \& Macdonald, A. J. (2009). Cytokines and IGF-I in delirious and non-delirious acutely ill older medical inpatients. Age Ageing, 38(3), 326-332.

Adamis, D., Treloar, A., Martin, F. C., Gregson, N., Hamilton, G., \& Macdonald, A. J. (2007). APOE and cytokines as biological markers for recovery of prevalent delirium in elderly medical inpatients. Int J Geriatr Psychiatry, 22(7), 688-694.

Al-Delaimy, W. K., von Muhlen, D., \& Barrett-Connor, E. (2009). Insulinlike growth factor-1, insulinlike growth factor binding protein-1, and cognitive function in older men and women. J Am Geriatr Soc, 57(8), 1441-1446.

Aleman, A., de Vries, W. R., de Haan, E. H., Verhaar, H. J., Samson, M. M., \& Koppeschaar, H. P. (2000). Age-sensitive cognitive function, growth hormone and insulin-like growth factor 1 plasma levels in healthy older men. Neuropsychobiology, 41(2), 73-78.

American Psychiatric Association. (1980). Diagnostic and Statistical Manual of Mental Disorder (3rd ed., text rev.). Washington, DC: American Psychiatric Association.

American Psychiatric Association. (2000). Diagnostic and Statistical Manual of 
Mental Disorder (4th ed., text rev.). Washington, DC: American Psychiatric Association.

Arwert, L. I., Deijen, J. B., \& Drent, M. L. (2005). The relation between insulin-like growth factor I levels and cognition in healthy elderly: a meta-analysis. Growth Horm IGF Res, 15(6), 416-422.

Borenstein, M., Hedges, L. V., Higgins, J. P., \& Rothstein, H. R. (2010). A basic introduction to fixed-effect and random-effects models for meta-analysis. Res Synth Methods, 1(2), 97-111.

Borenstein, M., Higgins, J. P., Hedges, L. V., \& Rothstein, H. R. (2017). Basics of meta-analysis: I2 is not an absolute measure of heterogeneity. Res Synth Methods, 8(1), 5-18.

Broadhurst, C., \& Wilson, K. (2001). Immunology of delirium: new opportunities for treatment and research. Br J Psychiatry, 179, 288-289.

Casserly, I., \& Topol, E. (2004). Convergence of atherosclerosis and Alzheimer's disease: inflammation, cholesterol, and misfolded proteins. Lancet, 363(9415), 1139-1146.

Cerejeira, J., Batista, P., Nogueira, V., Vaz-Serra, A., \& Mukaetova-Ladinska, E. B. (2013). The stress response to surgery and postoperative delirium: evidence of hypothalamic-pituitary-adrenal axis hyperresponsiveness and decreased 
suppression of the GH/IGF-1 Axis. J Geriatr Psychiatry Neurol, 26(3), 185-194.

Chu, C. S., Liang, C. K., Chou, M. Y., Lin, Y. T., Hsu, C. J., Chu, C. L., \& Chou, P. H. (2016). Lack of Association between Pre-Operative Insulin-Like Growth Factor-1 and the Risk of Post-Operative Delirium in Elderly Chinese Patients. Psychiatry Investig, 13(3), 327-332.

Cinar, M. A., Balikci, A., Sertoglu, E., Mehmet, A. K., Serdar, M. A., \& Ozmenler, K. N. (2014). Role of CRP, TNF-a, and IGF-1 in Delirium Pathophysiology. Noro Psikiyatr Ars, 51(4), 376-382.

D'Ercole, A. J., Ye, P., \& O'Kusky, J. R. (2002). Mutant mouse models of insulin-like growth factor actions in the central nervous system. Neuropeptides, 36(2-3), 209-220.

Duval, S., \& Tweedie, R. (2000). Trim and fill: A simple funnel-plot-based method of testing and adjusting for publication bias in meta-analysis. Biometrics, 56(2), 455-463.

Egberts, A., Wijnbeld, E. H., Fekkes, D., van der Ploeg, M. A., Ziere, G., Hooijkaas, H., . . Mattace-Raso, F. U. (2015). Neopterin: a potential biomarker for delirium in elderly patients. Dement Geriatr Cogn Disord, 39(1-2), 116-124.

Egger, M., Davey Smith, G., Schneider, M., \& Minder, C. (1997). Bias in 
meta-analysis detected by a simple, graphical test. BMJ, 315(7109), 629-634.

Ely, E. W., Inouye, S. K., Bernard, G. R., Gordon, S., Francis, J., May, L., . . D Dittus, R. (2001). Delirium in mechanically ventilated patients: validity and reliability of the confusion assessment method for the intensive care unit (CAM-ICU). JAMA, 286(21), 2703-2710.

Gluckman, P. D., Guan, J., Williams, C., Scheepens, A., Zhang, R., Bennet, L., \& Gunn, A. (1998). Asphyxial brain injury--the role of the IGF system. Mol Cell Endocrinol, 140(1-2), 95-99.

Godbout, J. P., \& Johnson, R. W. (2006). Age and neuroinflammation: a lifetime of psychoneuroimmune consequences. Neurol Clin, 24(3), 521-538.

Godbout, J. P., \& Johnson, R. W. (2009). Age and neuroinflammation: a lifetime of psychoneuroimmune consequences. Immunol Allergy Clin North Am, 29(2), 321-337.

Hammond, E. J., Meador, K. J., Aung-Din, R., \& Wilder, B. J. (1987). Cholinergic modulation of human P3 event-related potentials. Neurology, 37(2), 346-350.

Higgins, J. P., \& Green, S. (2011). Cochrane Handbook for Systematic Reviews of Interventions (5.1.0 ed.): The Cochrane Collaboration. Available from www.handbook.cochrane.org.

Higgins, J. P., \& Thompson, S. G. (2002). Quantifying heterogeneity in a 
meta-analysis. Stat Med, 21(11), 1539-1558.

Hughes, C. G., Patel, M. B., \& Pandharipande, P. P. (2012). Pathophysiology of acute brain dysfunction: what's the cause of all this confusion? Curr Opin Crit Care, $18(5), 518-526$.

Hustey, F. M., Meldon, S. W., Smith, M. D., \& Lex, C. K. (2003). The effect of mental status screening on the care of elderly emergency department patients. Ann Emerg Med, 41(5), 678-684.

Inouye, S. K., van Dyck, C. H., Alessi, C. A., Balkin, S., Siegal, A. P., \& Horwitz, R. I. (1990). Clarifying confusion: the confusion assessment method. A new method for detection of delirium. Ann Intern Med, 113(12), 941-948.

Jeppsson, B., Freund, H. R., Gimmon, Z., James, J. H., von Meyenfeldt, M. F., \& Fischer, J. E. (1981). Blood-brain barrier derangement in sepsis: cause of septic encephalopathy? Am J Surg, 141(1), 136-142.

Khan, B. A., Zawahiri, M., Campbell, N. L., \& Boustani, M. A. (2011). Biomarkers for delirium--a review. J Am Geriatr Soc, 59 Suppl 2, S256-261.

Kooijman, R., Sarre, S., Michotte, Y., \& De Keyser, J. (2009). Insulin-like growth factor I: a potential neuroprotective compound for the treatment of acute ischemic stroke? Stroke, 40(4), e83-88.

Lackey, B. R., Gray, S. L., \& Henricks, D. M. (2000). Actions and interactions of the 
IGF system in Alzheimer's disease: review and hypotheses. Growth Horm IGF Res, 10(1), 1-13.

Laurila, J. V., Pitkala, K. H., Strandberg, T. E., \& Tilvis, R. S. (2002). Confusion assessment method in the diagnostics of delirium among aged hospital patients: would it serve better in screening than as a diagnostic instrument? Int J Geriatr Psychiatry, 17(12), 1112-1119.

Le Roith, D. (2003). The insulin-like growth factor system. Exp Diabesity Res, 4(4), 205-212.

Lemstra, A. W., Kalisvaart, K. J., Vreeswijk, R., van Gool, W. A., \& Eikelenboom, P. (2008). Pre-operative inflammatory markers and the risk of postoperative delirium in elderly patients. Int J Geriatr Psychiatry, 23(9), 943-948.

Licastro, F., Candore, G., Lio, D., Porcellini, E., Colonna-Romano, G., Franceschi, C., \& Caruso, C. (2005). Innate immunity and inflammation in ageing: a key for understanding age-related diseases. Immun Ageing, $2,8$.

Meagher, D. J., Moran, M., Raju, B., Gibbons, D., Donnelly, S., Saunders, J., \& Trzepacz, P. T. (2007). Phenomenology of delirium. Assessment of 100 adult cases using standardised measures. Br J Psychiatry, 190, 135-141.

Morandi, A., Gunther, M. L., Pandharipande, P. P., Jackson, J. C., Thompson, J. L., Shintani, A. K., . . Girard, T. D. (2011). Insulin-like growth factor-1 and 
delirium in critically ill mechanically ventilated patients: a preliminary investigation. Int Psychogeriatr, 23(7), 1175-1181.

Murialdo, G., Barreca, A., Nobili, F., Rollero, A., Timossi, G., Gianelli, M. V., .. . Polleri, A. (2001). Relationships between cortisol, dehydroepiandrosterone sulphate and insulin-like growth factor-I system in dementia. J Endocrinol Invest, 24(3), 139-146.

Nasman, B., Olsson, T., Seckl, J. R., Eriksson, S., Viitanen, M., Bucht, G., \& Carlstrom, K. (1995). Abnormalities in adrenal androgens, but not of glucocorticoids, in early Alzheimer's disease. Psychoneuroendocrinology, 20(1), 83-94.

O'Keeffe, S. T., \& Gosney, M. A. (1997). Assessing attentiveness in older hospital patients: global assessment versus tests of attention. J Am Geriatr Soc, 45(4), 470-473.

Pai, J. K., Pischon, T., Ma, J., Manson, J. E., Hankinson, S. E., Joshipura, K., . . . Rimm, E. B. (2004). Inflammatory markers and the risk of coronary heart disease in men and women. $N$ Engl J Med, 351(25), 2599-2610.

Parrizas, M., \& LeRoith, D. (1997). Insulin-like growth factor-1 inhibition of apoptosis is associated with increased expression of the bcl-xL gene product. Endocrinology, 138(3), 1355-1358. 
Peruzzi, F., Prisco, M., Dews, M., Salomoni, P., Grassilli, E., Romano, G., .. .

Baserga, R. (1999). Multiple signaling pathways of the insulin-like growth factor 1 receptor in protection from apoptosis. Mol Cell Biol, 19(10), 7203-7215.

Radtke, F. M., Franck, M., MacGuill, M., Seeling, M., Lutz, A., Westhoff, S., .. . Spies, C. D. (2010). Duration of fluid fasting and choice of analgesic are modifiable factors for early postoperative delirium. Eur J Anaesthesiol, 27(5), 411-416.

Reinhardt, R. R., \& Bondy, C. A. (1994). Insulin-like growth factors cross the blood-brain barrier. Endocrinology, 135(5), 1753-1761.

Rudolph, J. L., \& Marcantonio, E. R. (2011). Review articles: postoperative delirium: acute change with long-term implications. Anesth Analg, 112(5), 1202-1211.

Sara, V. R., Hall, K., Enzell, K., Gardner, A., Morawski, R., \& Wetterberg, L. (1982). Somatomedins in aging and dementia disorders of the Alzheimer type. Neurobiol Aging, 3(2), 117-120.

Schmidt, R. E., Dorsey, D. A., Beaudet, L. N., Plurad, S. B., Parvin, C. A., \& Miller, M. S. (1999). Insulin-like growth factor I reverses experimental diabetic autonomic neuropathy. Am J Pathol, 155(5), 1651-1660.

Schuurmans, M. J., Shortridge-Baggett, L. M., \& Duursma, S. A. (2003). The 
Delirium Observation Screening Scale: a screening instrument for delirium. Res Theory Nurs Pract, 17(1), 31-50.

Sessler, C. N., Gosnell, M. S., Grap, M. J., Brophy, G. M., O'Neal, P. V., Keane, K. A., .. . Elswick, R. K. (2002). The Richmond Agitation-Sedation Scale: validity and reliability in adult intensive care unit patients. Am J Respir Crit Care Med, 166(10), 1338-1344.

Shen, H., Shao, Y., Chen, J., \& Guo, J. (2016). Insulin-Like Growth Factor-1, a Potential Predicative Biomarker for Postoperative Delirium Among Elderly Patients with Open Abdominal Surgery. Curr Pharm Des, 22(38), 5879-5883.

Steiner, L. A. (2011). Postoperative delirium. Part 1: pathophysiology and risk factors. Eur J Anaesthesiol, 28(9), 628-636.

Stroup, D. F., Berlin, J. A., Morton, S. C., Olkin, I., Williamson, G. D., Rennie, D., . . . Thacker, S. B. (2000). Meta-analysis of observational studies in epidemiology: a proposal for reporting. Meta-analysis Of Observational Studies in Epidemiology (MOOSE) group. JAMA, 283(15), 2008-2012.

Tham, A., Nordberg, A., Grissom, F. E., Carlsson-Skwirut, C., Viitanen, M., \& Sara, V. R. (1993). Insulin-like growth factors and insulin-like growth factor binding proteins in cerebrospinal fluid and serum of patients with dementia of the Alzheimer type. J Neural Transm Park Dis Dement Sect, 5(3), 165-176. 
Tobias, A. (1999). Assessing the influence of a single study in meta-analysis. STATA technical bulletin, 47, 15-17.

Torres-Aleman, I. (2010). Toward a comprehensive neurobiology of IGF-I. Dev Neurobiol, 70(5), 384-396.

Trejo, J. L., Carro, E., Garcia-Galloway, E., \& Torres-Aleman, I. (2004). Role of insulin-like growth factor I signaling in neurodegenerative diseases. $J \mathrm{Mol}$ Med (Berl), 82(3), 156-162.

Wells GA, S. B., O'Connell D, Peterson J, Welch V, et al.. (2011) The Newcastle-Ottawa Scale (NOS) for assessing the quality of nonrandomized studies in meta-analysis. Available:

www.ohri.ca/programs/clinical_epidemiology/oxford.asp.

Werner, H., \& LeRoith, D. (2014). Insulin and insulin-like growth factor receptors in the brain: physiological and pathological aspects. Eur Neuropsychopharmacol, 24(12), 1947-1953.

Wilson, K., Broadhurst, C., Diver, M., Jackson, M., \& Mottram, P. (2005). Plasma insulin growth factor-1 and incident delirium in older people. Int J Geriatr Psychiatry, 20(2), 154-159.

Witlox, J., Eurelings, L. S., de Jonghe, J. F., Kalisvaart, K. J., Eikelenboom, P., \& van Gool, W. A. (2010). Delirium in elderly patients and the risk of postdischarge 
mortality, institutionalization, and dementia: a meta-analysis. JAMA, 304(4), $443-451$.

Yen, T. E., Allen, J. C., Rivelli, S. K., Patterson, S. C., Metcalf, M. R., Flink, B. J., . . . Kwatra, M. M. (2016). Association between Serum IGF-I levels and Postoperative Delirium in Elderly Subjects Undergoing Elective Knee Arthroplasty. Sci Rep, 6, 20736.

Yudofsky, S. C., \& Hales, R. E. (2008). The American Psychiatric Publishing textbook of neuropsychiatry and behavioral neurosciences (5th ed.). Washington, DC: American Psychiatric Pub. 


\section{Table 1}

Summarization of recruited studies in current meta-analysis

\begin{tabular}{|c|c|c|c|c|c|c|c|}
\hline \multirow{3}{*}{$\begin{array}{l}\text { Author } \\
\text { (year) }\end{array}$} & \multirow{3}{*}{ Criteria } & \multirow{3}{*}{ Population or setting } & Delirium & \multicolumn{2}{|c|}{ Female } & \multirow{3}{*}{ IGF (ng/mL) } & \multirow{3}{*}{ Country } \\
\hline & & & & Mean age & Source & & \\
\hline & & & Non-delirium & & & & \\
\hline \multirow[t]{2}{*}{ Yen, T. E. } & \multirow[t]{2}{*}{$\mathrm{CAM}+$} & & \multicolumn{2}{|c|}{22} & \multicolumn{3}{|c|}{$57.0 \pm 18.6$} \\
\hline & & Elective knee arthroplasty & & $73.4 \pm 5.1$ & 52.0 serum & & USA \\
\hline (2016) & DSM-IV & & 76 & & & $68.4 \pm 31.6$ & \\
\hline \multirow[t]{2}{*}{ Shen, H. } & \multirow[t]{2}{*}{$\mathrm{CAM}+$} & & \multicolumn{2}{|l|}{36} & \multicolumn{3}{|c|}{$50.4 \pm 16.2$} \\
\hline & & Open abdominal surgery & & $70.1 \pm 6.4$ & 57.2 serum & & China \\
\hline (2016) & DSM-IV & & 104 & & & $67.0 \pm 24.1$ & \\
\hline \multirow[t]{2}{*}{ Chu, C. S. } & \multirow[t]{2}{*}{$\mathrm{CAM}+$} & & \multicolumn{2}{|l|}{23} & \multicolumn{3}{|c|}{$97.1 \pm 47.4$} \\
\hline & & Orthopedic surgery & & $81.7 \pm 4.0$ & 26.2 serum & & Taiwan \\
\hline (2016) & DSM-IV & & 80 & & & $96.1 \pm 47.6$ & \\
\hline Egberts, A. & DOSS + & Internal and geriatric ward & 23 & 82.6 & 53.5 serum & $* 48.1 \pm 32.1$ & Netherland \\
\hline
\end{tabular}




\begin{tabular}{|c|c|c|c|c|c|c|}
\hline (2015) & DSM-IV & & 63 & & & $71.0 \pm 43.5$ \\
\hline \multicolumn{2}{|l|}{ Cinar, M. A. } & & 15 & $64.2 \pm 10$ & & $1.44 \pm 1.76$ \\
\hline & DSM-IV & Cardiovascular surgery & & & 25.7 serum & Turkey \\
\hline (2014) & & & 20 & 3 & & $1.03 \pm 1.18$ \\
\hline \multirow[t]{2}{*}{ Cerejeira, J. } & $\mathrm{CAM}+$ & & 37 & & & $* 138.3 \pm 57.8$ \\
\hline & & Elective hip arthroplasty & & $73.0 \pm 6.3$ & 50.5 plasma & Portugal \\
\hline (2013) & DSM-IV & & 64 & & & $128.2 \pm 59.6$ \\
\hline Morandi, A. & $\mathrm{CAM}+$ & Critically ill mechanically ventilated & 86 & & & \\
\hline (2011) & CAM-ICU & patients & 24 & 65 & 48.2 serum & ${ }^{\#} \mathrm{n} / \mathrm{a}$ USA \\
\hline \multicolumn{7}{|l|}{ Lemstra, A. } \\
\hline & $\mathrm{CAM}+$ & & 18 & & & *109.9 \\
\hline W. & & Acute or elective hip surgery & & 78.9 & 69.1 blood & Netherland \\
\hline (2008) & DSM-IV & & 50 & & & 98.4 \\
\hline Adamis, D. & CAM & Elderly medical unit & 22 & $84.6 \pm 6.6$ & 67.1 serum & $55.8 \pm 33.7 \mathrm{UK}$ \\
\hline
\end{tabular}


Wilson, K. CAM +

Acute medical ward

(2005) DSM-III
12

84.5 $\pm 4.2 \quad 69.0$ plasma $\quad$ \# $\mathrm{n} / \mathrm{a}$ UK

*: nmol/L convert to $\mathrm{ng} / \mathrm{ml} \times 7.63$

${ }^{\#}$ : derived effect sizes from other statistical parameters

Abbreviation: CAM: confusion assessment method; DOSS: Delirium observation screening scale; ELISA: enzyme-linked immunosorbent assay; IGF: insulin-like growth factor; n/a: not available; RASS: Richmond agitation-sedation scale; RIA: radioimmunoassay; UK: United Kingdom; USA: United States of America. 
Records identified through database searching $(n=899)$
Additional records identified through other sources $(n=4)$

\section{Records identified ( $n=903)$}

Records after duplicates removed $(n=527)$

\section{Full-text articles assessed} for eligibility $(n=82)$

Studies included in qualitative synthesis $(n=10)$

\section{Ten studies included}

\section{Duplicated records excluded $(n=376)$}

\section{Excluded by title and abstract $(n=445)$}

Articles excluded $(n=72)$
(1) Meta-analysis
(2) Commentary
(3) Review
(4) The same sample sources
(5) Data from CSF
(6) Not about delirium
(7) Not about IGF-1 


\begin{tabular}{|c|c|c|c|c|}
\hline & $\begin{array}{r}\text { Hedges' }^{\prime} \\
g\end{array}$ & $\begin{array}{l}\text { Lower } \\
\text { limit }\end{array}$ & $\begin{array}{l}\text { Upper } \\
\text { limit }\end{array}$ & $p$-Value \\
\hline Adamis, D. (2007) & -0.512 & -1.046 & 0.023 & 0.061 \\
\hline Cerejeira, J. (2013) & 0.169 & -0.234 & 0.571 & 0.411 \\
\hline Chu, C.S. (2016) & 0.019 & -0.441 & 0.479 & 0.936 \\
\hline Cinar, M.A. (2014) & -0.260 & -0.917 & 0.397 & 0.437 \\
\hline Egberts, A. (2015) & -0.556 & -1.036 & -0.075 & 0.023 \\
\hline Lemstra, A.W. (2008) & 0.114 & -0.418 & 0.647 & 0.674 \\
\hline Morandi, A. (2011) & 0.009 & -0.441 & 0.458 & 0.970 \\
\hline Shen, H. (2016) & -0.738 & -1.125 & -0.352 & 0.000 \\
\hline Wilson, K. (2005) & -0.095 & -0.172 & -0.018 & 0.016 \\
\hline Yen, T.E. (2016) & -0.387 & -0.861 & 0.087 & 0.110 \\
\hline Overall & -0.209 & -0.393 & -0.026 & 0.025 \\
\hline
\end{tabular}

Overall

$-0.209$

$-0.393 \quad-0.026$

$\left.\right|_{-2.00}$
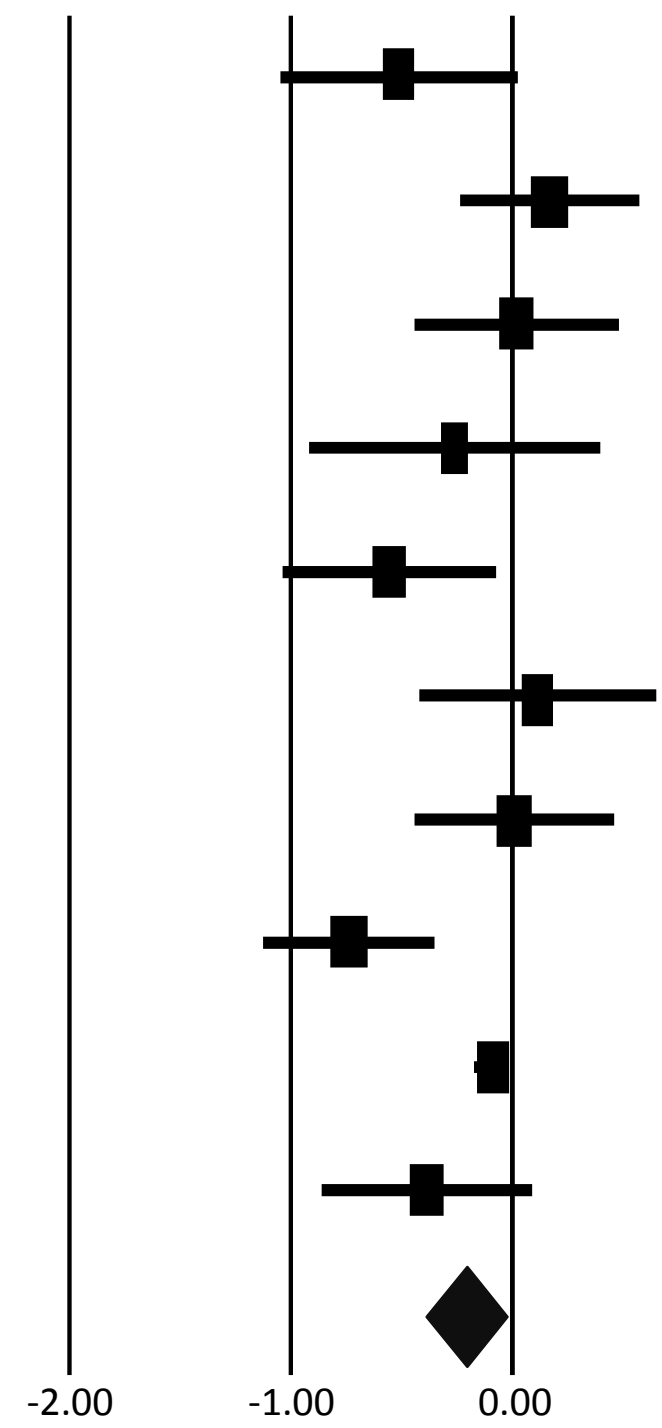

Lower IGF-1 in delirium Higher IGF-1 in delirium

Figure 2 Forest plot of results of pooled effect sizes of recruited studies 
Table 1

Summarization of recruited studies in current meta-analysis

\begin{tabular}{|c|c|c|c|c|c|c|}
\hline $\begin{array}{l}\text { Author } \\
\text { (year) }\end{array}$ & Criteria & Population or setting & $\begin{array}{l}\text { Delirium } \\
\text { Non-delirium }\end{array}$ & Mean age & $\begin{array}{l}\text { Female } \\
(\%)\end{array}$ & IGF (ng/mL) Country \\
\hline $\begin{array}{l}\text { Yen, T. E. } \\
(2016)\end{array}$ & $\begin{array}{l}\text { CAM + } \\
\text { DSM-IV }\end{array}$ & Elective knee arthroplasty & $\begin{array}{l}22 \\
76\end{array}$ & $73.4 \pm 5.1$ & 52.0 serum & $\begin{array}{l}57.0 \pm 18.6 \\
68.4 \pm 31.6\end{array}$ USA \\
\hline $\begin{array}{l}\text { Shen, H. } \\
\text { (2016) }\end{array}$ & $\begin{array}{l}\text { CAM + } \\
\text { DSM-IV }\end{array}$ & Open abdominal surgery & $\begin{array}{r}36 \\
104\end{array}$ & $70.1 \pm 6.4$ & 57.2 serum & $\begin{array}{l}50.4 \pm 16.2 \\
67.0 \pm 24.1\end{array}$ China \\
\hline $\begin{array}{l}\text { Chu, C. S. } \\
(2016)\end{array}$ & $\begin{array}{l}\text { CAM + } \\
\text { DSM-IV }\end{array}$ & Orthopedic surgery & $\begin{array}{l}23 \\
80\end{array}$ & $81.7 \pm 4.0$ & 26.2 serum & $\begin{array}{l}97.1 \pm 47.4 \\
96.1 \pm 47.6\end{array}$ Taiwan \\
\hline $\begin{array}{l}\text { Egberts, A. } \\
(2015)\end{array}$ & $\begin{array}{l}\text { DOSS + } \\
\text { DSM-IV }\end{array}$ & Internal and geriatric ward & $\begin{array}{l}23 \\
63\end{array}$ & 82.6 & 53.5 serum & $\begin{array}{c}* 48.1 \pm 32.1 \\
71.0 \pm 43.5\end{array}$ Netherland \\
\hline $\begin{array}{l}\text { Cinar, M. A. } \\
(2014)\end{array}$ & DSM-IV & Cardiovascular surgery & $\begin{array}{l}15 \\
20\end{array}$ & $\begin{array}{r}64.2 \pm 10 \\
3\end{array}$ & 25.7 serum & $\begin{array}{l}1.44 \pm 1.76 \\
1.03 \pm 1.18\end{array}$ Turkey \\
\hline $\begin{array}{l}\text { Cerejeira, J. } \\
(2013)\end{array}$ & $\begin{array}{l}\text { CAM + } \\
\text { DSM-IV }\end{array}$ & Elective hip arthroplasty & $\begin{array}{l}37 \\
64\end{array}$ & $73.0 \pm 6.3$ & 50.5 plasma & $\begin{array}{l}{ }^{*} 138.3 \pm 57.8 \\
128.2 \pm 59.6\end{array}$ Portugal \\
\hline $\begin{array}{l}\text { Morandi, A. } \\
(2011)\end{array}$ & $\begin{array}{l}\mathrm{CAM}+ \\
\mathrm{CAM}-\mathrm{ICU}\end{array}$ & $\begin{array}{l}\text { Critically ill mechanically ventilated } \\
\text { patients }\end{array}$ & $\begin{array}{l}86 \\
24\end{array}$ & 65 & 48.2 serum & ${ }^{\#} \mathrm{n} / \mathrm{a}$ USA \\
\hline $\begin{array}{l}\text { Lemstra, A. } \\
\text { W. } \\
(2008)\end{array}$ & $\begin{array}{l}\text { CAM + } \\
\text { DSM-IV }\end{array}$ & Acute or elective hip surgery & $\begin{array}{l}18 \\
50\end{array}$ & 78.9 & 69.1 blood & ${ }_{98.4}^{* 109.9}$ Netherland \\
\hline Adamis, D. & CAM & Elderly medical unit & 22 & $84.6 \pm 6.6$ & 67.1 serum & $55.8 \pm 33.7 \mathrm{UK}$ \\
\hline
\end{tabular}


(2007)

Wilson, $\mathrm{K} . \mathrm{CAM}+$

(2005) DSM-III
35

12
Acute medical ward

*: nmol/L convert to $\mathrm{ng} / \mathrm{ml} \times \mathrm{m} 7.63$

${ }^{\#}$ : derived effect sizes from other statistical parameters

Abbreviation: CAM: confusion assessment method; DOSS: Delirium observation screening scale; ELISA: enzyme-linked immunosorbent assay; IGF: insulin-like growth factor; n/a: not available; RASS: Richmond agitation-sedation scale; RIA: radioimmunoassay; UK: United Kingdom; USA: United States of America.
$75.2 \pm 33.7$

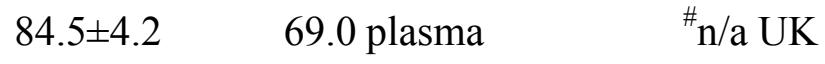


Supplemental table S1: MOOSE Checklist for Meta-analyses of Observational Studies

\begin{tabular}{clc}
\hline Item No & \multicolumn{1}{c}{ Recommendation } & $\begin{array}{c}\text { Reported on } \\
\text { Page No }\end{array}$ \\
\hline Reporting & of background should include & $6-7$ \\
1 & Problem definition & $6-7$ \\
2 & Hypothesis statement & $6-7$ \\
3 & Description of study outcome(s) & N/A \\
4 & Type of exposure or intervention used & $6-7$ \\
5 & Type of study designs used & $6-7$ \\
6 & Study population & \\
\hline
\end{tabular}

Reporting of search strategy should include

7 Qualifications of searchers (eg, librarians and investigators)

8 Search strategy, including time period included in the synthesis and key

9 Effort to include all available studies, including contact with authors

10 Databases and registries searched

8-10

11 Search software used, name and version, including special features used (eg, explosion)

N/A

12 Use of hand searching (eg, reference lists of obtained articles)

13 List of citations located and those excluded, including justification

Table 1, supplemental table S2

14 Method of addressing articles published in languages other than English

8-10

15 Method of handling abstracts and unpublished studies

16 Description of any contact with authors

$8-10$, acknowledgement

Reporting of methods should include

17 Description of relevance or appropriateness of studies assembled for assessing the hypothesis to be tested

18 Rationale for the selection and coding of data (eg, sound clinical principles or convenience)

19 Documentation of how data were classified and coded (eg, multiple raters, blinding and interrater reliability)

Assessment of confounding (eg, comparability of cases and controls in

20 studies where appropriate)

Assessment of study quality, including blinding of quality assessors,

21 stratification or regression on possible predictors of study results

22 Assessment of heterogeneity

Description of statistical methods (eg, complete description of fixed or

23 random effects models, justification of whether the chosen models account for predictors of study results, dose-response models, or cumulative metaanalysis) in sufficient detail to be replicated

24 Provision of appropriate tables and graphics

Table 1, Figure 2

Reporting of results should include

25 Graphic summarizing individual study estimates and overall estimate

Figure 2

26 Table giving descriptive information for each study included

Table 1, table 2

27 Results of sensitivity testing (eg, subgroup analysis) 
Reporting of discussion should include

29 Quantitative assessment of bias (eg, publication bias)

$16-17$

30 Justification for exclusion (eg, exclusion of non-English language citations)

$16-17$

supplementary

table S2

16-17,

31 Assessment of quality of included studies

Reporting of conclusions should include

32 Consideration of alternative explanations for observed results

Generalization of the conclusions (ie, appropriate for the data presented and

33 within the domain of the literature review)

34 Guidelines for future research

35 Disclosure of funding source

From: Stroup DF, Berlin JA, Morton SC, et al, for the Meta-analysis Of Observational Studies in Epidemiology (MOOSE) Group. Meta-analysis of Observational Studies in Epidemiology. A Proposal for Reporting. JAMA. 2000;283(15):2008-2012. doi: 10.1001/jama.283.15.2008.

Transcribed from the original paper within the NEUROSURGERY® Editorial Office, Atlanta, GA, United Sates. August 2012. 
Supplemental table S2: Excluded studies and reason

\section{$\underline{\text { Meta-analysis }(\mathrm{n}=1)}$}

Shi, C., Yang, C., Gao, R. and Yuan, W. (2015). Risk Factors for Delirium After Spinal Surgery: A Meta-Analysis. World Neurosurgery, 84, 1466-1472.

\section{Commentary $(\mathrm{n}=1)$}

Motosko, C., Brown, K. and Kwatra, M. (2012). Insulin-like growth factor 1 and delirium. Int Psychogeriatr, 24, 1872; author reply 1872-1873.

\section{$\underline{\text { Review }(\mathrm{n}=18)}$}

Adamis, D. and Meagher, D. (2011). Insulin-like growth factor I and the pathogenesis of delirium: a review of current evidence. J Aging Res, 2011, 951403.

Benhamou, D. and Brouquet, A. (2016). Postoperative cerebral dysfunction in the elderly: Diagnosis and prophylaxis. Journal of Visceral Surgery, 153, S27-S32.

Cerejeira, J., Firmino, H., Vaz-Serra, A. and Mukaetova-Ladinska, E. B. (2010). The neuroinflammatory hypothesis of delirium. Acta Neuropathol, 119, 737-754.

Cerejeira, J., Lagarto, L. and Mukaetova-Ladinska, E. B. (2014). The immunology of delirium. Neuroimmunomodulation, 21, 72-78.

Cunningham, C. and MacLullich, A. M. J. (2013). At the extreme end of the psychoneuroimmunological spectrum: Delirium as a maladaptive sickness behaviour response. Brain, Behavior, and Immunity, $28,1-13$.

de Rooij, S. E., van Munster, B. C., Korevaar, J. C. and Levi, M. (2007). Cytokines and acute phase response in delirium. Journal of Psychosomatic Research, 62, 521-525.

Fong, T. G., Davis, D., Growdon, M. E., Albuquerque, A. and Inouye, S. K. (2015). The interface between delirium and dementia in elderly adults. The Lancet Neurology, 14, 823-832.

Gaudreau, J.-D., Gagnon, P., Roy, M.-A., Harel, F. and Tremblay, A. (2005). Association Between Psychoactive Medications and Delirium in Hospitalized Patients: A Critical Review. Psychosomatics, 46, 302-316.

Gunther, M. L., Morandi, A. and Ely, E. W. (2008). Pathophysiology of Delirium in the Intensive Care Unit. Critical Care Clinics, 24, 45-65.

Khan, B. A., Zawahiri, M., Campbell, N. L. and Boustani, M. A. (2011). Biomarkers for delirium--a review. J Am Geriatr Soc, 59 Suppl 2, S256-261.

LeGrand, S. B. (2012). Delirium in Palliative Medicine: A Review. Journal of Pain and Symptom Management, 44, 583-594. 
Maldonado, J. R. (2008). Pathoetiological Model of Delirium: a Comprehensive Understanding of the Neurobiology of Delirium and an Evidence-Based Approach to Prevention and Treatment. Critical Care Clinics, 24, 789-856.

Maldonado, J. R. (2017). Acute Brain Failure: Pathophysiology, Diagnosis, Management, and Sequelae of Delirium. Critical Care Clinics, 33, 461-519.

Marcantonio, E. R., Rudolph, J. L., Culley, D., Crosby, G., Alsop, D. and Inouye, S. K. (2006). Serum biomarkers for delirium. J Gerontol A Biol Sci Med Sci, 61, 1281-1286.

Hall, R. J., Shenkin, S. D. and Maclullich, A. M. (2011). A systematic literature review of cerebrospinal fluid biomarkers in delirium. Dement Geriatr Cogn Disord, 32, 79-93.

Haymore, J. B. and Patel, N. (2016). Delirium in the Neuro Intensive Care Unit. Critical Care Nursing Clinics of North America, 28, 21-35.

Rizk, P., Morris, W., Oladeji, P. and Huo, M. (2016). Review of Postoperative Delirium in Geriatric Patients Undergoing Hip Surgery. Geriatr Orthop Surg Rehabil, 7, 100-105.

Simone, M. J. and Tan, Z. S. (2011). The role of inflammation in the pathogenesis of delirium and dementia in older adults: a review. CNS Neurosci Ther, 17, 506-513.

\section{The same sample sources $(\mathrm{n}=4)$}

Adamis, D., et al. (2009). Cytokines and IGF-I in delirious and non-delirious acutely ill older medical inpatients. Age Ageing, 38, 326-332; discussion 251.--> Same population from Adamis, D., Treloar, A., Martin, F.C., Gregson, N., Hamilton, G. and Macdonald, A. J. (2007). APOE and cytokines as biological markers for recovery of prevalent delirium in elderly medical inpatients. Int J Geriatr Psychiatry, 22, 688-694.

Adamis, D., Treloar, A., Gregson, N., Macdonald, A. J. and Martin, F. C. (2011). Delirium and the functional recovery of older medical inpatients after acute illness: the significance of biological factors. Arch Gerontol Geriatr, 52, 276-280. --> Same population from Adamis, D., Treloar, A., Martin, F. C., Gregson, N., Hamilton, G. and Macdonald, A. J. (2007). APOE and cytokines as biological markers for recovery of prevalent delirium in elderly medical inpatients. Int J Geriatr Psychiatry, 22, 688-694.

Adamis, D., et al. (2014). Phenomenological and biological correlates of improved cognitive function in hospitalized elderly medical inpatients. Arch Gerontol Geriatr, 59, 593-598. --> Same population from Adamis, D., Treloar, A., Martin, F. C., Gregson, N., Hamilton, G. and Macdonald, A.J. (2007). APOE and cytokines as biological markers for recovery of prevalent delirium in elderly medical inpatients. Int J Geriatr Psychiatry, 22, 688-694.

Kwatra, M. and Rivelli, S. (2008). Baseline Plasma Igf-1 Levels Relate to Postoperative Delirium in Knee Arthroplasty Patients. Orlando, FL: American Society of Anesthesiologists $\rightarrow$ Same population from Yen, T. E., et al. (2016). Association between Serum IGF-I levels and Postoperative Delirium in Elderly Subjects Undergoing Elective Knee Arthroplasty. Sci Rep, 6, 20736. 
Cape, E., et al. (2014). Cerebrospinal fluid markers of neuroinflammation in delirium: a role for interleukin-1beta in delirium after hip fracture. J Psychosom Res, 77, 219-225.

\section{$\underline{\text { Not about delirium }(\mathrm{n}=1)}$}

Cortes, E., et al. (2005). Insulin-like growth factor-1 gene splice variants as markers of muscle damage in levator ani muscle after the first vaginal delivery. American Journal of Obstetrics and Gynecology, 193, 64-70.

\section{$\underline{\text { No data about IGF-1 }(\mathrm{n}=46)}$}

Adamis, D., Treloar, A., Darwiche, F. Z., Gregson, N., Macdonald, A. J. and Martin, F. C. (2007). Associations of delirium with in-hospital and in 6-months mortality in elderly medical inpatients. Age Ageing, 36, 644-649.

Beloosesky, Y., Weiss, A., Hershkovitz, A., Hendel, D. and Barak, V. (2011). Serum transforming growth factor beta-1 post hip fracture repair in elderly patients. Cytokine, 54, 56-60.

Burkhart, C. S., et al. (2010). Modifiable and Nonmodifiable Risk Factors for Postoperative Delirium After Cardiac Surgery With Cardiopulmonary Bypass. Journal of Cardiothoracic and Vascular Anesthesia, 24, 555-559.

Burkhart, C. S., et al. (2014). Effect of n-3 fatty acids on markers of brain injury and incidence of sepsisassociated delirium in septic patients. Acta Anaesthesiol Scand, 58, 689-700.

Capri, M., et al. (2014). Pre-Operative, High-IL-6 Blood Level is a Risk Factor of Post-Operative Delirium Onset in Old Patients. Front Endocrinol (Lausanne), 5, 173.

Cerejeira, J., Nogueira, V., Luis, P., Vaz-Serra, A. and Mukaetova-Ladinska, E. B. (2012). The cholinergic system and inflammation: common pathways in delirium pathophysiology. $\mathrm{J}$ Am Geriatr Soc, 60, 669-675.

Chen, W., et al. (2017). Prevalence and risk factors for postoperative delirium in total joint arthroplasty patients: A prospective study. General Hospital Psychiatry, 46, 55-61.

Chen, X.-W., Shi, J.-W., Yang, P.-S. and Wu, Z.-Q. (2014). Preoperative plasma leptin levels predict delirium in elderly patients after hip fracture surgery. Peptides, 57, 31-35.

Fan, Y.-X., et al. (2014). Comparison of restrictive and liberal transfusion strategy on postoperative delirium in aged patients following total hip replacement: A preliminary study. Archives of Gerontology and Geriatrics, 59, 181-185.

Hall, R. J., et al. (2013). Delirium and Cerebrospinal Fluid S100B in Hip Fracture Patients: A Preliminary Study. The American Journal of Geriatric Psychiatry, 21, 1239-1243.

Hall, R. J., et al. (2015). Cerebrospinal fluid cortisol and cytokines in delirium after hip fracture. Psychoneuroendocrinology, 61, 49. 
Jackson, T. A., Sheehan, B., Maclullich, A. M., Gladman, J. R. and Lord, J. M. (2014). O2.02: Serum markers of inflammation and abnormalities of the HPA axis are not associated with poor outcome in delirium. European Geriatric Medicine, 5, Supplement 1, S55.

Jalleh, R., Koh, K., Choi, B., Liu, E., Maddison, J. and Hutchinson, M. R. (2012). Role of microglia and toll-like receptor 4 in the pathophysiology of delirium. Medical Hypotheses, 79, 735-739.

Kazmierski, J., Banys, A., Latek, J., Bourke, J. and Jaszewski, R. (2014). Raised IL-2 and TNF-alpha concentrations are associated with postoperative delirium in patients undergoing coronary-artery bypass graft surgery. Int Psychogeriatr, 26, 845-855.

Kazmierski, J., et al. (2014). Mild cognitive impairment with associated inflammatory and cortisol alterations as independent risk factor for postoperative delirium. Dement Geriatr Cogn Disord, 38, 65-78.

Li, G., Lei, X., Ai, C., Li, T. and Chen, Z. (2017). Low plasma leptin level at admission predicts delirium in critically ill patients: A prospective cohort study. Peptides, 93, 27-32.

Lin, S.-M., et al. (2008). Risk factors for the development of early-onset delirium and the subsequent clinical outcome in mechanically ventilated patients. Journal of Critical Care, 23, 372-379.

Liu, P., et al. (2013). High serum interleukin-6 level is associated with increased risk of delirium in elderly patients after noncardiac surgery: a prospective cohort study. Chin Med J (Engl), 126, 36213627.

Macdonald, A., Adamis, D., Treloar, A., and Martin, F. (2007). C-reactive protein levels predict the incidence of delirium and recovery from it. Age Ageing. 2007, 36, 222-5.

MacLullich, A. M., et al. (2011). Cerebrospinal fluid interleukin-8 levels are higher in people with hip fracture with perioperative delirium than in controls. J Am Geriatr Soc, 59, 1151-1153.

Mariscalco, G., et al. (2012). Preoperative Statin Therapy Is Not Associated With a Decrease in the Incidence of Delirium After Cardiac Operations. The Annals of Thoracic Surgery, 93, 1439-1447.

Morandi, A., et al. (2013). Vitamin D and delirium in critically ill patients: a preliminary investigation. Journal of Critical Care, 28, 230-235.

Mossello, E., et al. (2014). O2.04: Predictors and prognosis of delirium among older subjects in Cardiology Intensive Care Units. European Geriatric Medicine, 5, Supplement 1, S55.

Naeije, G., Depondt, C., Meeus, C., Korpak, K., Pepersack, T. and Legros, B. (2014). EEG patterns compatible with nonconvulsive status epilepticus are common in elderly patients with delirium: a prospective study with continuous EEG monitoring. Epilepsy \& Behavior, 36, 18-21.

Neerland, B. E., et al. (2016). Associations Between Delirium and Preoperative Cerebrospinal Fluid CReactive Protein, Interleukin-6, and Interleukin-6 Receptor in Individuals with Acute Hip Fracture. J Am Geriatr Soc, 64, 1456-1463.

Ngo, L. H., et al. (2017). Methodologic considerations in the design and analysis of nested case-control studies: association between cytokines and postoperative delirium. BMC Med Res Methodol, 17, 88 . 
Nguyen, D. N., Huyghens, L., Schiettecatte, J., Smitz, J. and Vincent, J.-L. (2016). High prolactin levels are associated with more delirium in septic patients. Journal of Critical Care, 33, 56-61.

O'Sullivan, R., Inouye, S. K. and Meagher, D. (2014). Delirium and depression: inter-relationship and clinical overlap in elderly people. The Lancet Psychiatry, 1, 303-311.

Pearson, A., et al. (2010). Cerebrospinal fluid cortisol levels are higher in patients with delirium versus controls. BMC Res Notes, 3, 33.

Plaschke, K., et al. (2010). Early postoperative delirium after open-heart cardiac surgery is associated with decreased bispectral EEG and increased cortisol and interleukin-6. Intensive Care Med, 36, 2081-2089.

Rudolph, J. L., et al. (2008). Chemokines are associated with delirium after cardiac surgery. J Gerontol A Biol Sci Med Sci, 63, 184-189.

Schmitt, E. M., et al. (2012). Novel risk markers and long-term outcomes of delirium: the successful aging after elective surgery (SAGES) study design and methods. J Am Med Dir Assoc, 13, 818.e811-810.

Skrede, K., Wyller, T. B., Watne, L. O., Seljeflot, I. and Juliebo, V. (2015). Is there a role for monocyte chemoattractant protein-1 in delirium? Novel observations in elderly hip fracture patients. BMC Res Notes, 8, 186.

Sun, L., et al. (2016). Production of inflammatory cytokines, cortisol, and Abeta1-40 in elderly oral cancer patients with postoperative delirium. Neuropsychiatr Dis Treat, 12, 2789-2795.

Tsang, L. F., et al. (2012). Developing a predictive tool for post-operative delirium in orthopaedic settings in Hong Kong. International Journal of Orthopaedic and Trauma Nursing, 16, 147-159.

Tsuruta, R., et al. (2010). Prevalence and associated factors for delirium in critically ill patients at a Japanese intensive care unit. General Hospital Psychiatry, 32, 607-611.

van den Boogaard, M., et al. (2011). Biomarkers associated with delirium in critically ill patients and their relation with long-term subjective cognitive dysfunction; indications for different pathways governing delirium in inflamed and noninflamed patients. Crit Care, 15, R297.

van Munster, B. C., et al. (2010). Cortisol, interleukins and S100B in delirium in the elderly. Brain and Cognition, 74, 18-23.

van Munster, B. C., Korevaar, J. C., Zwinderman, A. H., Levi, M., Wiersinga, W. J. and De Rooij, S. E. (2008). Time-course of cytokines during delirium in elderly patients with hip fractures. J Am Geriatr Soc, 56, 1704-1709.

van Munster, B. C., et al. (2012). Longitudinal assessment of serum anticholinergic activity in delirium of the elderly. J Psychiatr Res, 46, 1339-1345.

van Steijn, J.H., Nieboer, P., Hospers, G.A., de Vries, E.G., and Mulder, N.H. (2001). Delirium after interleukin-2 and alpha-interferon therapy for renal cell carcinoma. Anticancer Res, 21, 3699-700.

Vasunilashorn, S. M., et al. (2015). Cytokines and Postoperative Delirium in Older Patients Undergoing Major Elective Surgery. J Gerontol A Biol Sci Med Sci, 70, 1289-1295. 
Westhoff, D., et al. (2013). Preoperative cerebrospinal fluid cytokine levels and the risk of postoperative delirium in elderly hip fracture patients. J Neuroinflammation, 10, 122.

Westhoff, D., et al. (2015). Preoperative protein profiles in cerebrospinal fluid in elderly hip fracture patients at risk for delirium: A proteomics and validation study. BBA Clinical, 4, 115-122.

Zhang, Z., Pan, L., Deng, H., Ni, H. and Xu, X. (2014). Prediction of delirium in critically ill patients with elevated C-reactive protein. Journal of Critical Care, 29, 88-92.

Zhang, Z.-Y., et al. (2016). Impact of length of red blood cells transfusion on postoperative delirium in elderly patients undergoing hip fracture surgery: A cohort study. Injury, 47, 408-412. 
Supplemental Table S3: Newcastle-Ottawa Quality Assessment Scale (NOS) scores of included studies

\begin{tabular}{lcccr}
\hline Author (year) & Selection & Comparability & Outcome & Total scores \\
\hline Yen, T. E. (2016) & 4 & 2 & 2 & 8 \\
Shen, H. (2016) & 4 & 2 & 1 & 7 \\
Chu, C. S. (2016) & 4 & 1 & 2 & 7 \\
Egberts, A. (2015) & 4 & 1 & 2 & 8 \\
Cinar, M. A. (2014) & 4 & 1 & 2 & 7 \\
Cerejeira, J. (2013) & 4 & 2 & 2 & 8 \\
Morandi, A. (2011) & 4 & 1 & 2 & 7 \\
Lemstra, A. W. (2008) & 4 & 2 & 2 & 7 \\
Adamis, D. (2007) & 4 & 2 & 1 & \\
Wilson, K. (2005) & 4 & & & 7 \\
\hline
\end{tabular}


supplement figure (IGF OR IGF1 protein $\mathrm{OR}$ inflammation mediators OR insulin-like growth factor OR cytokine) AND (delirium OR acute confusion OR consciousness disturbance)

Limits: $\mathrm{n} / \mathrm{a}$ Date: $2017 / 10 / 2$ ( $n=292)$
Embase (IGF OR IGF1 protein OR inflammation mediators OR insulin-like growth factor $O R$ cytokine) AND (delirium OR acute confusion OR consciousness disturbance) Limits: $n / a$ Date: $2017 / 10 / 2$ $(n=28)$

\section{ScienceDirect \\ ClinicalKey}

(insulin-like growth factor) AND (delirium) Limits: $n / a$

Date:

2017/10/2 $(n=207)$ (insulin-like growth factor) AND (delirium) Limits: $n / a$

Date:

2017/10/2 $(n=160)$
Cochrane Library ProQuest (IGF OR IGF1 protein $\mathrm{OR}$ inflammation mediators OR insulin-like growth factor $O R$ cytokine) AND (delirium OR acute confusion OR consciousness disturbance) Limits: $n / a$ Date: $2017 / 10 / 2$ $(n=27)$
(IGF OR IGF1 protein OR inflammation mediators OR insulin-like growth factor OR cytokine) AND (delirium OR acute confusion OR consciousness disturbance) Limits: $n / a$ Date: $2017 / 10 / 2$ $(n=183)$
ClinicalTrials.gov (IGF OR IGF1 protein $\mathrm{OR}$ inflammation mediators OR insulin-like growth factor OR cytokine) AND (delirium OR acute confusion OR consciousness disturbance) Limits: $\mathrm{n} / \mathrm{a}$ Date: $2017 / 10 / 2$ $(n=2)$

$\mid$

Excluded articles $(n=445)$

Not meeting inclusion criteria $(n=72)$

Included articles in meta-analysis

$(n=10)$
Supplement figure S1 Detailed search strings used for the electronic database search and results 